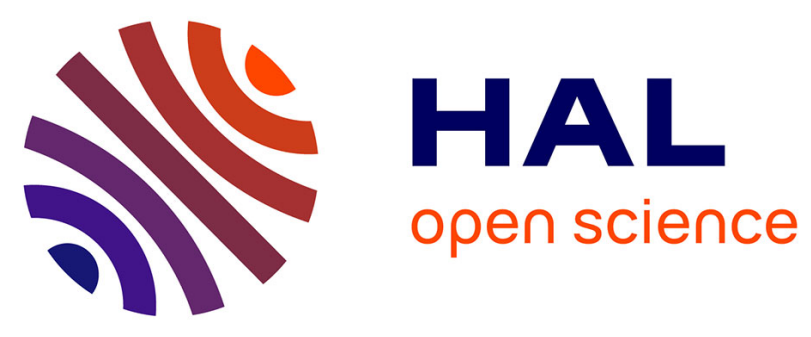

\title{
Human body impact on mobile visible light communication link
}

Clément Le Bas, Stéphanie Sahuguede, Anne Julien-Vergonjanne, Abdeslam Behlouli, Pierre Combeau, Lilian Aveneau

\section{To cite this version:}

Clément Le Bas, Stéphanie Sahuguede, Anne Julien-Vergonjanne, Abdeslam Behlouli, Pierre Combeau, et al.. Human body impact on mobile visible light communication link . 10th International Symposium on Communication Systems, Networks and Digital Signal Processing (CSNDSP 2016), Jul 2016, Prague, Czech Republic. 10.1109/CSNDSP.2016.7573900 . hal-01493236

\section{HAL Id: hal-01493236 \\ https://hal-unilim.archives-ouvertes.fr/hal-01493236}

Submitted on 21 Mar 2017

HAL is a multi-disciplinary open access archive for the deposit and dissemination of scientific research documents, whether they are published or not. The documents may come from teaching and research institutions in France or abroad, or from public or private research centers.
L'archive ouverte pluridisciplinaire HAL, est destinée au dépôt et à la diffusion de documents scientifiques de niveau recherche, publiés ou non, émanant des établissements d'enseignement et de recherche français ou étrangers, des laboratoires publics ou privés. 


\section{Human body impact on mobile visible light communication link}

Conference Paper · July 2016

DOI: 10.1109/CSNDSP.2016.7573900

CITATIONS

0

6 authors, including:

\section{Stephanie Sahuguede}

University of Limoges

48 PUBLICATIONS 72 CITATIONS

SEE PROFILE

\section{Abdeslam Behlouli}

XLIM Research Institute

6 PUBLICATIONS 9 CITATIONS

SEE PROFILE
READS

40

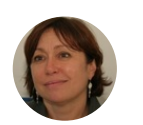

Anne Julien-Vergonjanne

University of Limoges

138 PUBLICATIONS 258 CITATIONS

SEE PROFILE

Lilian Aveneau

XLIM Research Institute

70 PUBLICATIONS 213 CITATIONS

SEE PROFILE

Some of the authors of this publication are also working on these related projects: 


\section{Human Body Impact on Mobile Visible Light Communication Link}

\author{
C. Le Bas, S. Sahuguede, A. Julien-Vergonjanne \\ XLIM UMR CNRS 7252/Labex Sigma-Lim \\ University of Limoges \\ France
}

\begin{abstract}
Shadowing impact for indoor mobile visible light communications (VLC), is linked to the person wearing the optical receiver, depending on the body features and varying according the data rate. Here, we investigate VLC performance considering two body models with different reflectivity values. In addition, we consider a mobile receiver with random orientations to account body movements. Results show that for low data rates, a $2 \mathrm{D}$ body model with perpendicular receiver orientation allows suitable performance estimation. For high data rates, $2 \mathrm{D}$ body model can be used but link efficiency is deteriorated depending on receiver orientation variations whatever body reflectivity values are.
\end{abstract}

Keywords-Visible Light Communication, Mobile indoor optics, Channel characterizations, Body model

\section{INTRODUCTION}

As energy saving is a topic of interest, much research on Visible Light Communications (VLC) based on Light Emitting Diodes (LEDs) has been developed in recent years [1-3]. LEDs have several attractive characteristics, so they are used in many contexts in order to replace classic lighting methods like incandescent light. Actually, they are energy efficient, and they can be easily combined to perform dimming for brightness, warm management or cool lighting. In addition, LEDs structure allows fast switching abilities, so they emerge to be the best candidates for VLC networking, which main goal is to provide both illumination and communication.

VLC and more generally Optical Wireless Communications (OWC) have a number of advantages over existing wireless communication ones and constitute a solution to overcome spectrum congestion of radio frequency (RF) communications.

First, OWC spectral domain offers a high and not regulated bandwidth. In addition, optical components are cheap so it is possible to deploy a low cost network. Furthermore, this technology does not generate electromagnetic interferences, so optical network will not interfere with RF existing ones, making its application particularly promising in sensitive environment like hospitals or airplanes [4,5].

Several outdoor applications of OWC have been considered such as intra and extra vehicular communications or to be a complement to RF in the 5G technology [6]. Nevertheless, it is for indoor applications that much research was conducted and

\author{
A. Behlouli, P. Combeau, L. Aveneau \\ XLIM UMR CNRS 7252/ Labex Sigma-Lim \\ University of Poitiers \\ France
}

a first standard, IEEE 802.15.7, has been recently proposed, allowing regulating the use of VLC [7].

For indoor environments, the high power provided by the room lighting allows obtaining high Signal to Noise Ratios (SNR), increasing the interest of this kind of scenario. In addition, due to the light confinement, transmitted data are restricted to room area, contributing to information security enhancement.

Most current indoor VLC studies consider a fixed receiver with emitters placed on the ceiling. These configurations offer a high quality of service (QoS) but it has been shown that shadowing effect due to the presence of objects and/or moving person can degrade VLC performance $[1,8,9]$.

In addition, when the receiver is mobile, for example worn by a person, the body movements induce receiver orientation variations which can also contribute to performance degradation because of link blocking cases. Methods like angle diversity can overcome this problem $[10,11]$ but it is necessary to have a more complex receiver. This is difficult in the case of a system worn by a person.

In this paper, we study a classical VLC configuration constituted of several emitters fixed on the room celling but assuming a mobile and simple receiver worn by a moving person. Our contribution is to determine body impact on the performance including orientation and position variations of the receiver. The analysis is provided thanks to ray-tracing channel model with different body profiles and considering body/room reflectivity features. Performance is obtained as a function of the data rate.

The paper is organized as follows: in part II we describe the studied environment and the different body models used in our simulation. Part III presents the results obtained taking into account body impact. Finally, conclusions are given in part IV.

\section{DESCRIPTION OF THE STUDIED SYSTEM}

\section{A. System Overview}

The configuration studied in this paper has been considered in many VLC studies [12-16]. As seen in Fig. 1, it consists in an empty room $(5 \mathrm{mx} 5 \mathrm{mx} 3 \mathrm{~m})$, where four VLC transmitters, each constituted of 3600 LEDs, are placed on the celling 
(coordinates are indicated in TABLE I). The total emitted power is $144 \mathrm{~W}$ and ensures a minimum of 400 lux in the room [15].The receiver can be at any position on a $2 \mathrm{D}$ plan at $1.2 \mathrm{~m}$ from the floor. The walls and the ceiling are considered as plaster surfaces and the floor as plastic one.

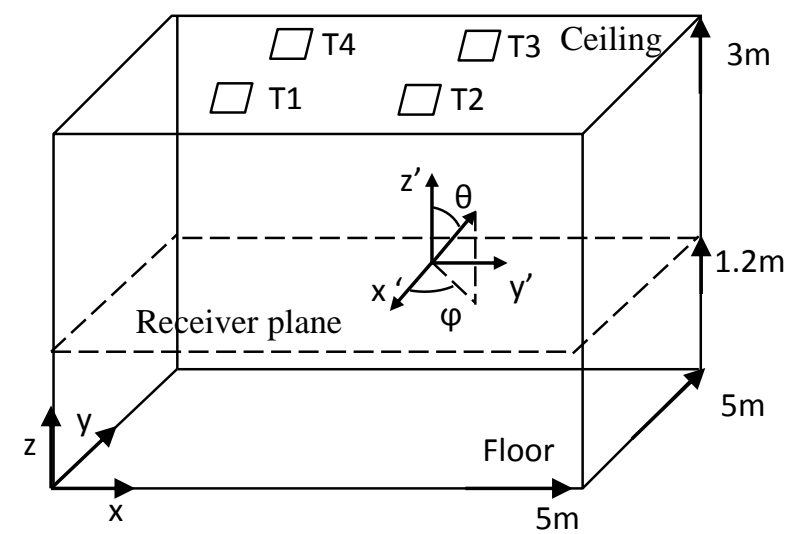

Fig. 1. VLC scenario configuration

Moreover, we assume a random uniform distribution of receiver positions within the $2 \mathrm{D}$ plan in order to model the receiver mobility. As mobility also implies the variations of the receiver orientations (for example due to the body movements), we define azimuthal and longitudinal angles respectively $\varphi$ and $\theta$, as illustrated in Fig. 1, whose values are uniformly and randomly chosen within $\varphi \in[0,2 \pi]$ and $\theta \in$ $\left[0, \frac{\pi}{3}\right]$.

TABLE I. Configuration parameters

T1: [ $\left.\begin{array}{llll}1,25 & 1.25 & 3\end{array}\right]$

Coordinates $[\mathrm{x}$ y $\mathrm{z}](\mathrm{m})$

T2 : [1.25 3.75 3]

T3 : [3.75 3.753$]$

$\mathrm{T} 4$ : [ [ $\left.\begin{array}{lll}3.75 & 1.25 & 3\end{array}\right]$

\begin{tabular}{ccc} 
Transmitters & $\begin{array}{c}\text { Half angle of power } \\
\text { Orientation }\end{array}$ & $60^{\circ}$ \\
& $\begin{array}{c}\text { Numbers of LEDs per } \\
\text { array }\end{array}$ & $60 * 60$ \\
& $\begin{array}{c}\text { Average transmitted } \\
\text { power (per LEDs) } P_{L E D}\end{array}$ & $10 \mathrm{~mW}$ \\
\hline \hline \multirow{2}{*}{ Receiver } & Responsivity & $0.28 \mathrm{~A} / \mathrm{W}$ \\
& Field of View & $65^{\circ}$
\end{tabular}

\section{B. Channel model}

Usually, studied VLC configurations are static considering a receiver oriented towards the ceiling $(\theta=0$ in Fig. 1). In this case, the VLC channel is mainly characterised by Line Of Sight (LOS) optical paths.

However, in indoor environment, it is also important to take into account optical reflections from surfaces which can impact the received power. As we consider receiver mobility and variations of receiver orientation, this is all the more significant. The global channel impulse response can be expressed as:

$$
h_{\text {total }}(t)=\sum_{i=1}^{N} h_{i}(t)=\sum_{i=1}^{N} \sum_{k=0}^{\infty} h_{i}^{(k)}(t)
$$

Where $N$ is the number of sources and $h_{i}^{(k)}(t)$ is the $k$ bounces contribution to the channel impulse response according to the $i^{\text {th }}$ source. It has been shown that a suitable model for indoor dynamic VLC channel can be obtained considering three reflections $(\mathrm{k}=3)$ of the optical signal [14].

An accurate way to calculate $h_{\text {total }}(t)$ uses a ray tracing algorithm relying on the Monte Carlo method. For this aim, we use a ray-based simulator developed at the XLIM Laboratory, which reduces computation time by implementing some techniques of variance reduction such as importance sampling or next event estimation methods [17].

For our simulation set-up, each of the $N$ impulse responses is determined over duration of $60 \mathrm{~ns}$ with a step of $0.15 \mathrm{~ns}$ and 2 million launched rays.

Various environmental elements such as walls and objects contribute to the optical reflections. The human body, which can wear the receiver device, can also have a great influence on the propagation loss.

In this paper, to study the impact of the human body on the VLC performance, we have to model its geometry on one side and its reflectance properties on the other side.

A first question concerns the level of details to be used by simulation and its impact on impulse response. To answer this question, we consider two geometrical models, one in 2D corresponding to the profile of a generic human body ( $c f$. Fig. 2 (a)), and another one being a highly detailed 3D mesh [18] ( $c f$. Fig. 2 (b)). These two models represent a body of $1.72 \mathrm{~m}$ height and $45 \mathrm{~cm}$ width. The $3 \mathrm{D}$ one presents a thickness of $25 \mathrm{~cm}$ and is composed of 1796 facets (triangles).

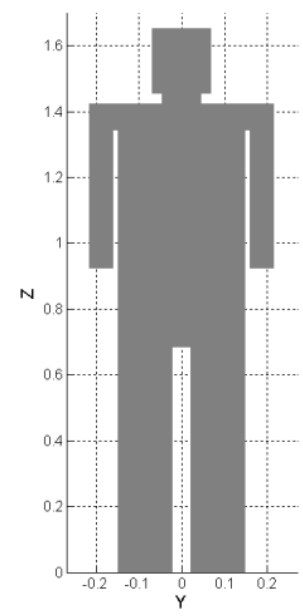

(a)

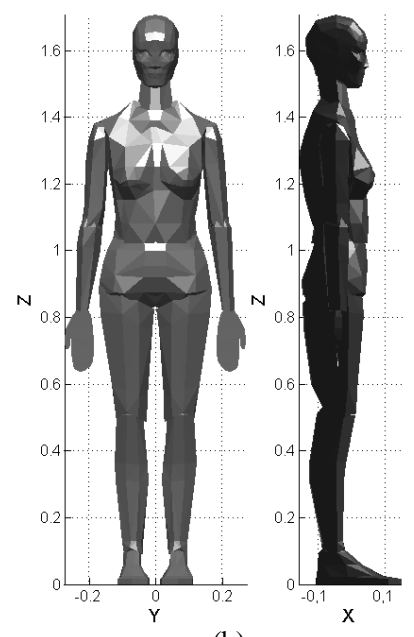

(b)
Fig. 2. Geometrical models of the body in (a) 2D, (b) 3D

The second question concerns the reflectance properties of the environment. Each facet composing the body (2D or 3D) is characterized by a Lambertian Bidirectional Reflectance Distribution Function (BRDF) [18] which is a purely diffuse model. This one is defined by a single parameter $\rho$, 
corresponding to the reflectivity at normal incidence. The value of $\rho$ varies from 0 to 1 . A weak value represents very absorbing surfaces, while a high value is associated to very shiny materials. The surface of a human body exposed to light propagation is composed of two main parts, the skin and the clothes, and their $\rho$ values vary according to the light wavelength. Fig. 3 resumes the corresponding range of some $\rho$ values according to the light wavelength [19].

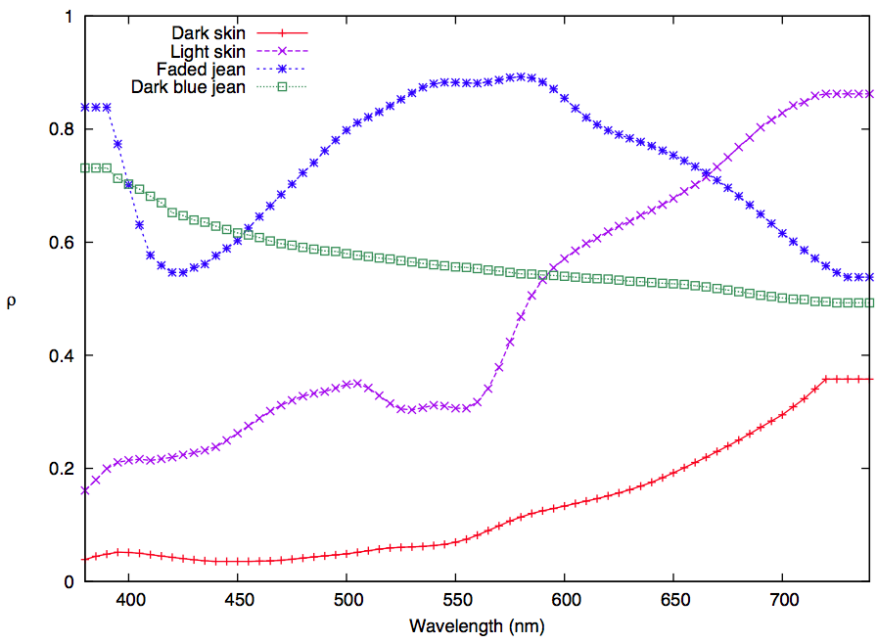

Fig. 3. Evolution of some $\rho$ values according to the light wavelength

On one side, white human skin $\rho$ values go from 0.16 to 0.86 when dark skin values go from 0.04 to 0.35 . The corresponding mean values are 0.51 and 0.19 respectively. On the other side, dark blue jeans $\rho$ values go from 0.5 to 0.75 which correspond to a mean value of 0.62 , while faded jeans $\rho$ values go from 0.55 to 0.72 with a mean value of 0.63 .

In conclusion, to take into account the whole range of $\rho$ values connected to the different human skins and clothes, and so to obtain the most representative conclusions, all light simulations presented in this paper are obtained concerning two extreme mean values of $\square$, respectively $\square_{\square}=0.1$ and $\square_{\square}=0.7$.

Finally, the reflectance properties of the rest of the environment (walls, ceiling and floor) are presented in the TABLE II. They have been fixed according to [20].

TABLE II. Reflectance properties of the room

\begin{tabular}{ccc}
\hline \hline $\begin{array}{c}\text { Geometrical } \\
\text { elements }\end{array}$ & BRDF & $\rho$ values \\
\hline \hline Walls/Ceiling & Lambertian & 0.73 \\
Floor & Lambertian & 0.35 \\
\hline \hline
\end{tabular}

\section{HUMAN BODY IMPACT}

\section{A. Impact on the optical gain}

For the simulation we have considered 961 receiver positions uniformly chosen within the $2 \mathrm{D}$ plane. In addition the receiver is worn by a person whose body is modelled by the 2D and 3D previously described shapes and supposed to be moving with the receiver. So, we have assumed that the body is oriented towards the receiver, and located at $20 \mathrm{~cm}$ of it. At last, for the orientation variations, 18 values for each receiver position have been simulated. They have been chosen uniformly within the interval defined in Part.II A.

The probability density function (PDF) of the optical gain $H(0)$ considering five different scenarios is reported in Fig. 4 and 5. The optical gain $H(0)$ is defined as:

$$
H(0)=\int_{-\infty}^{+\infty} h_{\text {total }}(t) d t
$$

The studied scenarios consider receiver mobility. This means that we simulate 961 receiver positions and that receiver orientation varies as defined in section II:

- (a): mobile receiver without considering body

- $\quad$ (b): mobile receiver and 2D body model with $\square_{\square}=0.7$

- $\quad$ (c): mobile receiver and 3D body model with $\square_{\square}=0.7$

- $\quad$ (d): mobile receiver and 2D body model with $\square_{\square}=0.1$

- $\quad$ (e): mobile receiver and 3D body model with $\square_{\square}=0.1$

First, it may be observed by comparing scenario (b) and (c) in Fig. 4 and scenario (d) and (e) in Fig. 5, that for a given value of reflectivity coefficient, the results obtained with $2 \mathrm{D}$ and $3 \mathrm{D}$ body models are quite similar. This means that it is not necessary to use a complex model which is a significant achievement given the diversity of human body.

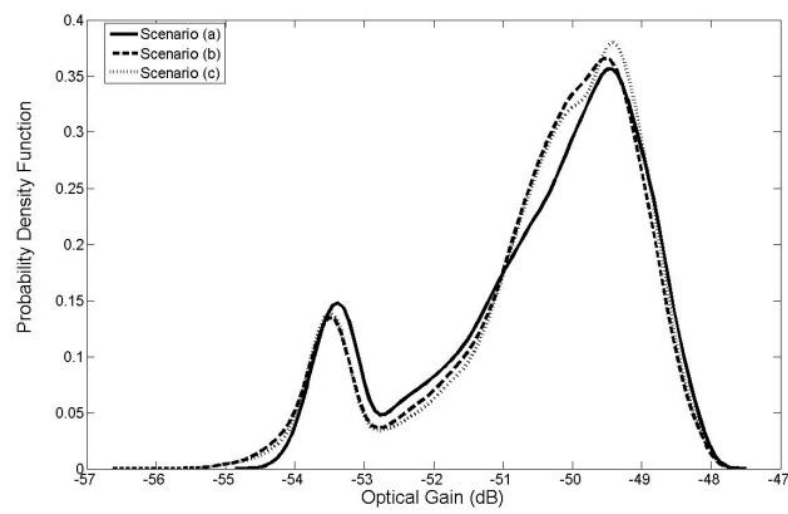

Fig. 4. Probability density function of the optical gain for scenarios (a), (b) and (c)

Then, if we compare scenario (a) and (b) in Fig. 4, it can be noted that as expected, a surface with a high value of $\rho$ doesn't significantly modify the optical gain distribution. On the contrary, we can observe that results for a weak $\rho$ value in Fig. 5 exhibit different conclusions. Indeed, the optical gain PDF is more spread of about $5 \mathrm{~dB}$ in scenarios (d) and (e) compared to scenario (a) where the body is not taken into account. Those results show that the body has an impact on the optical gain, mostly depending on its $\rho$ value and not on its shape. To discuss performance, we will use in the following a $2 \mathrm{D}$ model with the two $\square \square \square$ extreme values $\square_{\square}$ respectively $\square_{\square \square}=0.1$ and $\square_{\square}=0.7$. 


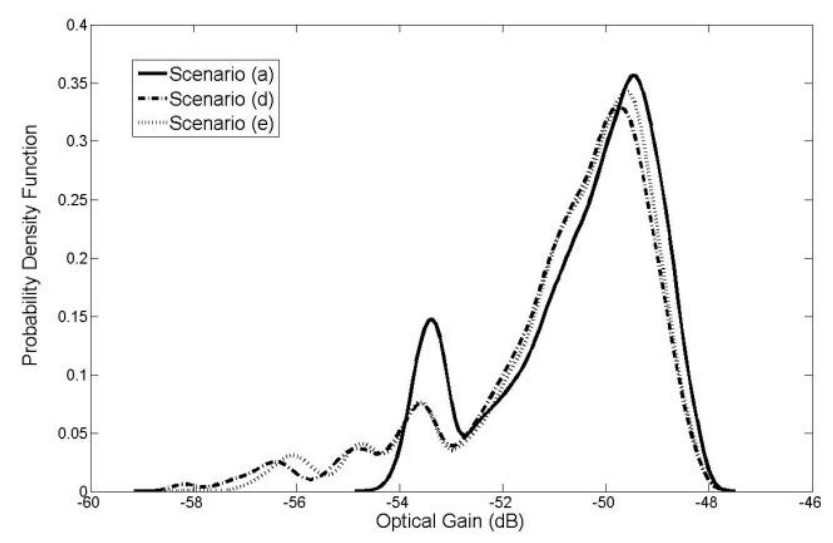

Fig. 5. Probability density function of the optical gain for scenarios (a), (d) and (e)

\section{B. Impact on the channel performance}

To establish the performance, we first determine the SNR for the scenarios (a), (b) and (d). In the case of On-Off Keying modulation (OOK) the SNR can be expressed as [12]:

$$
S N R_{O O K}=\frac{2\left(R \cdot P_{r-s i g}\right)^{2}}{\sigma_{\text {shot }}^{2}+\sigma_{\text {thermal }}^{2}}
$$

Where $R$ is the photodetector sensitivity, $\sigma_{\text {shot }}^{2}$ represents the shot noise power and $\sigma_{\text {thermal }}^{2}$ the thermal one. In our case, we consider that $\sigma_{\text {thermal }}^{2}$ is very small compared to $\sigma_{\text {shot }}^{2}$. So, the noise power is obtained as:

$$
\sigma_{\text {shot }}^{2}=2 q R\left(P_{r-s i g}+P_{r-i s i}\right) B+2 q I_{B} I_{2} B
$$

Where $q$ is the electron quantum charge, $I_{B}$ is the photocurrent due to background radiations, $I_{2}$ is the bandwidth noise factor and $B=1 / T$ is the bandwidth of the signal. We set $I_{2}=0.562$ and $I_{B}=5100 \mu \mathrm{A}[21] . P_{r-s i g}$ and $P_{r-i s i}$ are respectively the received power within the symbol duration $T$ and the power linked to Inter Symbol Interference (ISI). They are defined as:

$$
\begin{aligned}
& P_{r-s i g}=\int_{0}^{T} \sum_{i=1}^{N} h_{i}(t) \otimes x(t) d t \\
& P_{r-i s i}=\int_{T}^{\infty} \sum_{i=1}^{N} h_{i}(t) \otimes x(t) d t
\end{aligned}
$$

$x(t)$ is the transmitted optical pulse. The symbol $\otimes$ denotes the convolution. Also, the average transmitted power $P_{t}$ is given by:

$$
P_{t}=\lim _{T \rightarrow \infty} \frac{1}{2 T} \int_{-T}^{+T} x(t) d t
$$

We use the outage probability criterion to discuss the performance considering mobility [18]. It corresponds to the probability that the communication link would not ensure an aimed Quality of Service (QoS) defined here by a targeted SNR value named $S N R_{0}$ related to the BER for OOK modulation by:

$$
B E R=\frac{1}{2} \operatorname{erfc}\left(\sqrt{\frac{S N R_{0}}{2}}\right)
$$

The outage probability $P_{\text {out }}$ can be expressed as:

$$
P_{\text {out }}=P_{r}\left(S N R<S N R_{0}\right)
$$

We have reported in Fig. 6 and Fig. 7 the outage probability for two data rates: $R_{b 1}=1 \mathrm{Mbps}$ and $\mathrm{R}_{\mathrm{b} 2}=350 \mathrm{Mbps}$ respectively for scenarios (a);(b) and scenarios (a);(d).

First, we can see that to achieve a $\mathrm{P}_{\text {out }}$ of $10^{-1}$, the impact of the body is negligible whatever the data rate and the body reflectivity are.

Secondly, for lower values of $P_{\text {out }}$, the results show that body impact is more significant when the data rate increases. For example in Fig. 6, for a $P_{\text {out }}$ of $10^{-3}$, we have determined the corresponding $S N R_{0}$ value for each scenario.

The difference in terms of SNR is $\triangle \mathrm{SNR}=0.4 \mathrm{~dB}$ between scenario (a) and (b) for $R_{b 1}$ whereas $\Delta S N R=21 d B$ for $R_{b 2}$. In addition, this effect is all the more significant as the reflectivity value is weak: in Fig. $7, \Delta \mathrm{SNR}=8 \mathrm{~dB}$ between scenario (a) and (d) for $\mathrm{R}_{\mathrm{b} 1}$ and $\Delta \mathrm{SNR}=36 \mathrm{~dB}$ for $\mathrm{R}_{\mathrm{b} 2}$.

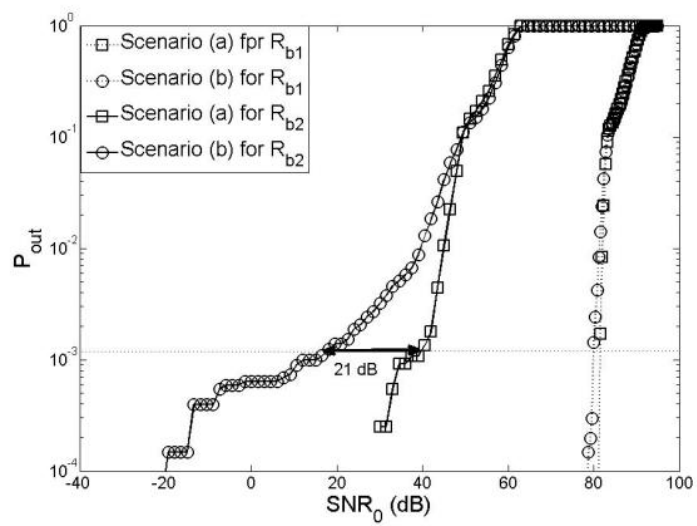

Fig. 6. Outage probability for $\mathrm{R}_{\mathrm{b} 1}=1 \mathrm{Mbps}$ and $\mathrm{R}_{\mathrm{b} 2}=350 \mathrm{Mbps}$ considering $2 \mathrm{D}$ body and $\rho_{2}=0.7$

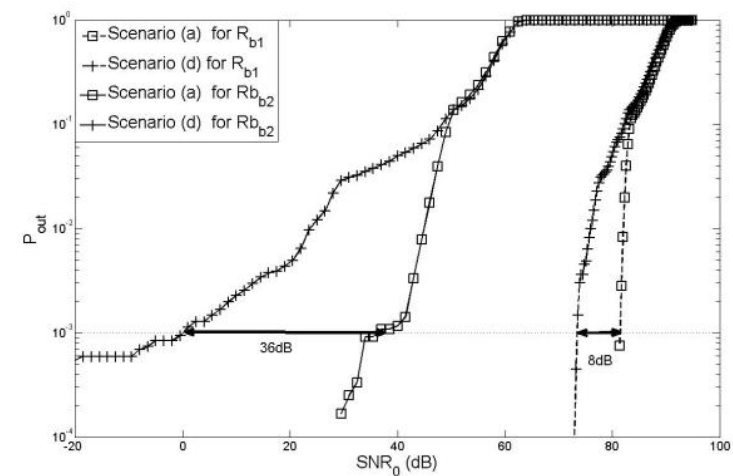

Fig. 7. Outage probability for $R_{b 1}=1 \mathrm{Mbps}$ and $R_{b 2}=350 \mathrm{Mbps}$ considering $2 \mathrm{D}$ body and $\rho_{1}=0.1$

Nevertheless, the body shape and $\rho$ value are not the only parameters having penalizing effect. Indeed, we have already shown in [6] for the same VLC configuration that receiver 
orientation variations have a strong impact on the performance. But, this had been performed without considering body.

So, in order to determine the impact of orientation variations with a body, we have considered another scenario (f) and compared the results with scenario (b):

- (f) mobile receiver with 2D body model with $\square_{\square}=0.7$ and fixed receiver orientation $(\theta=0)$

We have reported in Fig. 8 the outage probability for $R_{b 1}$ and $\mathrm{R}_{\mathrm{b} 2}$ for the two scenarios (b) and (f). It can be observed that the achievable performance between both scenarios at $\mathrm{R}_{\mathrm{b} 1}$ are similar, meaning that for low data rates, considering a $2 \mathrm{D}$ body with only perpendicular receiver orientation is an assumption allowing suitable performance estimation.

However, for high data rates, the impact of the receiver orientation variations cannot be neglected. Actually, performance with $R_{b 2}$ is highly degraded in scenario (f) compared to (b). For example, for a $P_{\text {out }}$ of $10^{-3}$, the $\Delta$ SNR value between scenario (b) and (f) is quite equal to $30 \mathrm{~dB}$. This high value of $\triangle$ SNR can be explained by the fact that considering a strong ISI because of high data rates implies cases in some orientations where the received signal is almost blocked by the body and so the optical gain is quite equal to zero. This blocking effect has been observed considering a high body $\rho_{2}$ value $\left(\rho_{2}=\right.$ 0.7 ) and it is likely that the effect will be even more significant for smaller values.

To investigate body blocking impact for high data rates regarding body $\rho$ value, we thus consider the two extreme values $\rho_{1}$ and $\rho_{2}$ and that receiver orientations randomly vary. We have reported in Fig. 9, the evolution of the minimal $S N R_{0}$ value required for an outage probability of $10^{-3}$ and a wide range of data rate from $1 \mathrm{kbps}$ to $500 \mathrm{Mbps}$. The studied scenarios are (a), (b) and (d).

As expected, we can observe that for a high $\rho$ value (scenario (b)) and for data rates lower than 250Mbps, the impact of the body on the $S N R_{0}$ value is not significant. However, from $300 \mathrm{Mbps}$, the $S N R_{0}$ value rapidly decreases and so performance is degraded. As we have seen above, this is linked to ISI impact in some orientations.

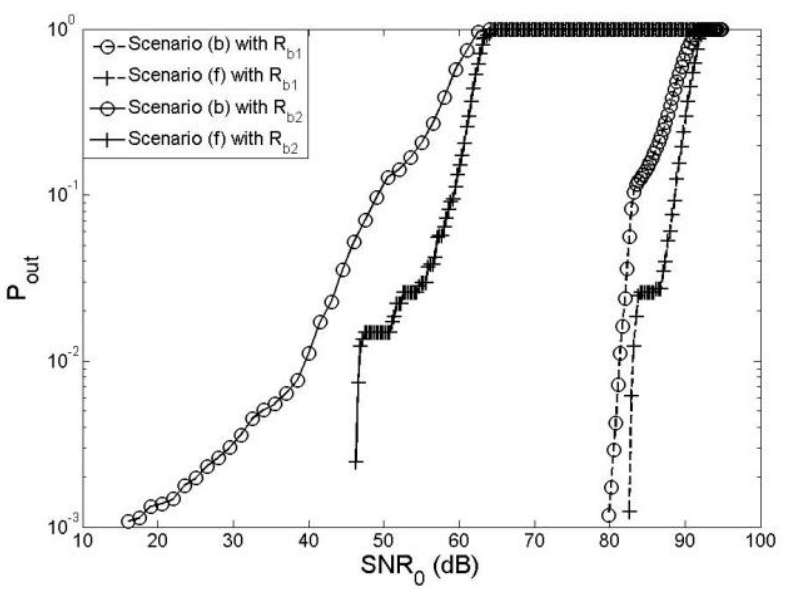

Fig. 8. Outage probability for scenarios (b) and (f) for $R_{b 1}=1 M b p s$ and $R_{b 2}$ $=350 \mathrm{Mbps}$
For a weak $\rho$ value in case (d), the $S N R_{0}$ value is always lower than in case (b) even for low data rates. For example, for $\mathrm{R}_{\mathrm{b}}=100 \mathrm{Mbps}$, the $S N R_{0}$ gap between the two cases is around $\triangle \mathrm{SNR}=15.3 \mathrm{~dB}$. However, until around $300 \mathrm{Mbps}$ the requested $S N R_{0}$ values for the targeted $P_{\text {out }}$ are still high enough to ensure low BER values with OOK modulation.

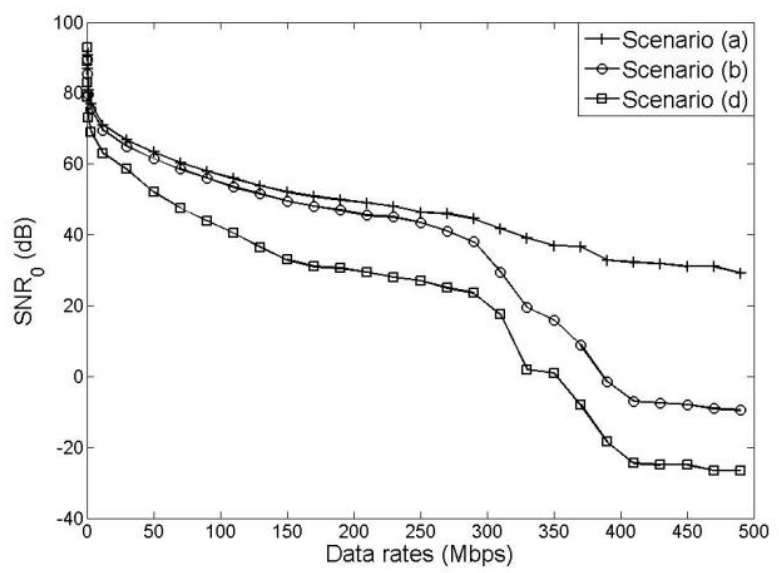

Fig. 9. Minimal $S N R$ value at $P_{\text {out }}=10^{-3}$ for data rates from $1 \mathrm{kbps}$ to 450 Mbps for scenarios (a), (b) and (d)

Finally, from $300 \mathrm{Mbps}$ we can also remark as previously that the $S N R_{0}$ values highly decrease. This means that to design an efficient high speed VLC link it is important to model the presence of the body along with receiver orientations to take into account blocking cases.

These results confirm that the choice of the body's $\rho$ value is very important, because regarding its value the body can or cannot have an impact on the channel performance, even at low data rates.

\section{CONCLUSION}

The main goal of this paper was to investigate the impact of the body on a VLC channel link. This channel was considered mobile, meaning that positions and orientations of the receiver are randomly varying. Moreover, we have considered that the receiver is worn by a person, whose body is also taken into account. Our study was based on the comparison of two body models: one in $2 \mathrm{D}$ and the other one in $3 \mathrm{D}$. We have also considered two extreme $\rho$ values for the body $\left(\rho_{1}=0.1\right.$ and $\rho_{2}=$ $0.7)$.

First, by comparing the VLC gain distributions obtained with the two body models, we have shown that the body shape does not have a significant impact whatever the reflectivity is. Thus, it is not necessary to use a complex model to study the body impact on the VLC link performance.

Performance has been then analyzed in terms of outage probability considering different values of data rates corresponding to different ISI cases. We have shown that to achieve low QoS (outage probability of $10^{-1}$ ) body impact is not significant whatever the data rate or the body reflectivity are. 
However, for high QoS (outage probability of $10^{-3}$ ), performance is degraded depending on the body $\rho$ value along with the data rate one.

In addition, we have included the receiver orientation impact in our analysis. The results have first shown that for low data rates, considering only perpendicular receiver orientation is an assumption allowing suitable performance estimation. But, for high data rates, it is necessary to model receiver orientation variations along with the body features.

Finally, we have evaluated the minimal SNR value required for a given outage probability for a wide range of data rates (1kbps to $450 \mathrm{Mbps}$ ).

We have seen that for a high body $\rho$ value and data rates lower than $300 \mathrm{Mbps}$, body impact is not significant, whereas from higher data rates performance can be highly and rapidly degraded due to body blocking impact. For weak body reflectivity values, the results are similar but more penalizing.

Those results have been obtained for a specific environment, but they highlight that the impact of the body on a mobile VLC channel depends on the data rate and on the $\rho$ value of the body. This is a first study which has to be completed in particular by studying the impact on the performance of the distance between the receiver and the body.

\section{REFERENCES}

[1] Chvojka, P.; Zvanovec, S.; Haigh, P.A.; Ghassemlooy, Z., "Channel Characteristics of Visible Light Communications Within Dynamic Indoor Environment," Lightwave Technology, Journal of , vol.33, no.9, pp.1719,1725, May1, 12015

[2] Shlomi A., "Visible Light Communication," Cambridge University Press, February 2015

[3] H. Elgala, R. Mesleh, and H. Haas, "Indoor Optical Wireless Communication: Potential and State-of-the-Art," IEEE Communications Magazine, vol. 49, no. 9, 2011

[4] Murai, R.; Sakai, T.; Kawano, H.; Matsukawa, Y.; Kitano, Y.; Honda, Y.; Campbell, K.C., "A novel visible light communication system for enhanced control of autonomous delivery robots in a hospital," System Integration (SII), 2012 IEEE/SICE International Symposium on , vol., no., pp.510,516, 16-18 Dec. 2012

[5] Chan, E., "Wireless optical links for airplane applications," Photonics Society Summer Topical Meeting Series, 2012 IEEE , vol., no., pp.76,77, 9-11 July 2012

[6] Pergoloni, S.; Biagi, M.; Colonnese, S.; Cusani, R.; Scarano, G., "Coverage optimization of 5G atto-cells for visible light communications access," in Measurements \& Networking $(M \& N), 2015$ IEEE International Workshop on , vol., no., pp.1-5, 12-13 Oct. 2015

[7] Boucouvalas, A.; Chatzimisios, P.; Ghassemlooy, Z.; Uysal, M.; Yiannopoulos, K., "Standards for indoor Optical Wireless Communications," Communications Magazine, IEEE , vol.53, no.3, pp.24,31, March 2015

[8] T. Komine and M. Nakagawa, "A study of shadowing on indoor visible light wireless communication utilizing plural white LED lightings," in Proc. 1st Int. Symp. Wireless Commun. Syst., Sep. 20-22, 2004, pp. 3640.

[9] R. C. Kizilirmak, "Impact of repeaters on the performance of indoor visible light communications", Turk J Elec Eng \& Comp Sci (2015) 23: $1159-1172$

[10] Hussein, AT and Elmirghani, J (2015) 10 Gbps Mobile Visible Light Communication System Employing Angle Diversity, Imaging Receivers, and Relay Nodes. IEEE/OSA Journal of Optical Communications and Networking, 7 (8). 718 - 735. ISSN 1943-0620

[11] A. Nuwanpriya, H. Siu-Wai, C. S. Chen, "Indoor MIMO Visible Light Communications: Novel Angle Diversity Receivers for Mobile Users," IEEE Journal on Selected Areas in Communications, Institute of Electrical and Electronics Engineers, 2015, pp.1-13
[12] Z. Ghassemlooy, W. Popoola, S. Rajbhandari, "Opitcal Wireless Communications System and Channel Modelling with MATLAB" Northumbia University, August 2012, pp. 462-464

[13] Grubor, J.; Randel, S.; Langer, K.-D.; Walewski, J., "Bandwidth-efficient indoor optical wireless communications with white light-emitting diodes," in Communication Systems, Networks and Digital Signal Processing, 2008. CNSDSP 2008. 6th International Symposium on, vol., no., pp.165-169, 25-25 July 2008

[14] Le Bas, C.; Sahuguede, S.; Julien-Vergonjanne, A.; Behlouli, A.; Combeau, P.; Aveneau, L., "Impact of receiver orientation and position on Visible Light Communication link performance," in Optical Wireless Communications (IWOW), 2015 4th International Workshop on , vol., no., pp.1-5, 7-8 Sept. 2015

[15] J.J. Tan, C.Q. Zou, S.H. Du, J.T. Tan, "Simulation of MIMO channel characteristics for indoor visible light communication with LEDs", Optik - International Journal for Light and Electron Optics, June 2013

[16] Barry, J.R.; Kahn, J.M.; Krause, W.J.; Lee, E.A.; Messerschmitt, D.G., "Simulation of multipath impulse response for indoor wireless optical channels," Selected Areas in Communications, IEEE Journal on, vol.11, no.3, pp.367,379, Apr 1993

[17] A. Behlouli, P. Combeau, L. Aveneau, S. Sahuguède and A. JulienVergonjanne, 'Efficient simulation of optical wireless channel, Application to WBANs with MISO link', Procedia Computer Science, vol. 40, pp 190-197(2014)

[18] http://www.robots.ox.ac.uk/ wmayol/3D/nancy_matlab.html

[19] A.S. Glassner, "Principles of Digital Image Synthesis", Morgan Kaufmann Publishers Inc., 1994.

[20] Kwonhyung Lee; Hyuncheol Park; Barry, J.R., "Indoor Channel Characteristics for Visible Light Communications," Communications Letters, IEEE, vol.15, no.2, pp.217,219, February 2011

[21] A.J. Moreira, Rui T. Valadas and A.M. de Oliveira Duarte, "Optical interference produced by artificial light", Wireless Networks 3 (1997) $\underline{131-140}$ 